\title{
Low-dose computed tomography in assessment of pulmonary abnormalities in children with febrile neutropenia suffering from malignant diseases
}

\author{
Urszula Zaleska-Dorobisz 1, A, D, F, Cyprian Olchowy 1, B, E, Mateusz Łasecki1, C, Dąbrówka Sokołowska-Dąbek¹, A, D, \\ Aleksander Pawluś' ${ }^{1, C}$, , Jowita Frączkiewicz ${ }^{2, B, C, E}$, Ewa Gorczyńska ${ }^{2, A, B}$ \\ ${ }^{1}$ Department of Radiology, Wroclaw Medical University, Poland \\ 2 Department of Pediatric Stem Cell Transplantation, Hematology and Oncology, Wroclaw Medical University, Poland \\ A - research concept and design; B - collection and/or assembly of data; C - data analysis and interpretation; \\ $D$ - writing the article; $E$ - critical revision of the article; $F$ - final approval of article
}

Address for correspondence

Cyprian Olchowy

E-mail: cyprian.olchowy@gmail.com

Funding sources

None declared

Conflict of interest

None declared

Received on September 30, 2016

Revised on December 14, 2016

Accepted on January 05,2017

DOI

\section{Abstract}

Background. Management of febrile neutropenia in pediatric patients is challenging. Chest X-ray and CT scan help to identify infective foci; however, exposure to radiation is a risk factor for development of secondary cancer. For this reason, attention is paid to reducing radiation exposure.

Objectives. The aim of the study was to define the role of LDCT examination in the early detection of pulmonary lesions in children during oncology or autoimmune treatment complicated by neutropenia-related fever. Additionally, we focused on the possibility to optimize image quality in low-dose protocols.

Material and methods. The study included 138 pediatric patients (mean age 8.08 years) with fever of $38.2^{\circ} \mathrm{C}$ or higher with an absolute neutrophil count of $10 \mathrm{~mm}$ with or without surrounding $\mathrm{GGO}$ or cavitations was sensitive at $77 \%$ and specific at $65 \%$ for fungal infection insert after neutrophil count: $<500 / p L$ who underwent chest X-ray and LDCT in the maximal interval of $24 \mathrm{~h}$. CT findings were compared with initial and final diagnosis as well as with clinical information.

Results. LDCT detected pulmonary abnormalities in 116 patients (84.06\%) showing ground-glass opacities (GG0) ( $n=79$ ), nodules $(n=60)$ and air-space consolidations $(n=58)$. Radiologists correctly diagnosed infective lesions in 94 out of 116 patients (81.03\%). The presence of random or pleural-based nodules. Diagnosis of pyogenic infection based on the presence of air-space consolidation, pleural effusion, GGO or centrilobular nodules showed a sensitivity of $78 \%$ and specificity of $67 \%$, whereas patchy or diffuse GGO, interstitial thickening and/or air-space consolidation showed a high sensitivity of $81 \%$ and specificity of $68 \%$ for Pneumocystis jirovecii pneumonia.

Conclusions. LDCT is an excellent modality in the diagnostic algorithm in patients with febrile neutropenia. It allows early detection and detailed characterization of pulmonary abnormalities. Using contrast, unenhanced CT examinations can further reduce radiation dose and diminish the number of complications without a negative influence on the diagnostic process.

Key words: febrile neutropenia, pulmonary infection, low dose CT, pediatric neoplasms, hematologic diseases

$10.17219 /$ acem/68292

\section{Copyright}

Copyright by Author(s)

This is an article distributed under the terms of the

Creative Commons Attribution Non-Commercial License

(http://creativecommons.org/licenses/by-nc-nd/4.0/) 
High-dose combination therapy in cancer patients may lead to side effects which require rapid diagnosis and adequate treatment. One of the most common complications resulting from myelosuppression is neutropenia rooted in the use of cytotoxic or immunosuppressive polytherapy for malignant or autoimmune diseases. Bacterial pathogens, mainly gram-positive aerobes, are responsible for approximately $90 \%$ of infections in the early phase of neutropenia. ${ }^{1,2}$ Overall, the mortality rate in neutropenic patients varies between 8 and 21\% depending on the specific neoplastic disease, patients' general condition, type of infectious complication and presence of other diseases. The main risk factors for febrile neutropenia among oncology patients are cytotoxic chemotherapy and steroids as well as bacterial, viral or fungal infections. Neutropenia-related fever has been also described in pediatric non-HIV-infected patients. ${ }^{3,4}$

The precise identification of the pathogen is often difficult. First, a rising number of infections are polymicrobial, resulting in difficulties in choosing specific treatment which produce good outcomes and few side effects. Secondly, broad spectrum antibiotics predispose to superadded fungal infections. And thirdly, many infections mimic graft-versus-host disease, radiation toxicity, drug toxicity or even pulmonary hemorrhage, which delays proper diagnosis and treatment.

The clinical course of pulmonary complications in children with febrile neutropenia is usually severe. In addition to some typical symptoms including sudden onset and dyspnea, signs of peritoneal irritation in the case of abdominal involvement, a septic fever, neurological dysfunctions, pleural or peritoneal effusion and paralytic ileus may be observed. An early and correct diagnosis is critical to introduce proper treatment and avoid surgical procedures. The basic examination during diagnosis of pulmonary pathologies is still a standard chest X-ray, then thoracic computed tomography (CT). Both X-ray and CT of the chest can help in early detection of an infective focus and its presumptive characterization, as advances in diagnostic imaging have significantly improved visualization of the lungs and mediastinal structures on CT images. Although multisector CT is more accurate than chest radiography, the higher radiation dose imparted with standard-dose thin-section CT is a major limitation. ${ }^{5}$ A high dose of radiation has the potential to cause cancer, particularly in children, who have a greater risk per unit dose, when multiple scans are required. The radiosensitivity in children is widely discussed. Current estimations show that about $25 \%$ of cancer types occurring in children are more susceptible than in adults. Additionally, a one-year-old child is as much as 10 times more susceptible to radiation-induced cancer than an adult. ${ }^{6,7}$

CT has become a frequent and essential first-use diagnostic modality. It not only detects the abnormality with a high degree of sensitivity and specificity, but also differentiates between types of infection and between infectious and noninfectious etiologies. It can detect pulmonary infiltrates at an earlier stage than a chest X-ray while awaiting the micro- biological report. ${ }^{3}$ Because of this increased use of CT, delivering the same quality imaging while keeping radiation dose to an absolute minimum has become an important feature of the diagnostic process and has led to the development of several low-dose computed tomography (LDCT) protocols. In our study, we aimed to define the role of LDCT examination in the early detection and characterization of pulmonary infections and the differential diagnosis of respiratory system diseases in children during oncology or autoimmune treatment complicated by neutropenia-related fever.

\section{Material and methods}

The study group consisted of 138 patients (49 girls and 89 boys) with age range from 9 months to 18 years (mean 8.08; median 7) with febrile neutropenia in the course of the malignancy process treated in the Department and Clinic of Pediatric Oncology, Hematology and Bone Marrow Transplantation in Wrocław between November $1^{\text {st }}$, 2011 and June $23^{\text {rd }}, 2014$. The most frequent oncologic diagnoses were acute lymphocytic leukemia (ALL), acute myelogenous leukemia (AML) and neuroblastoma. The characteristics of the oncologic diseases in the study group are summarized in Table 1.

All of the children included presented respiratory problems such as cough, dyspnea, respiratory distress or pleural pain. Other nonspecific symptoms, including vomiting, diarrhea, obstruction and gastrointestinal infection, were reported by 32 subjects (23.19\%). Acute abdominal pain occurred in 34 patients (24.64\%).

Every patient was tested for basic hematological markers: hemoglobin $(\mathrm{g} / \mathrm{dL})$, red and white blood cell count, granulocytes, lymphocytes and platelet count. Febrile neutropenia in children was defined as a temperature rise up to $38.2^{\circ} \mathrm{C}$ or more with an ANC of $500 / \mathrm{pL}$ or less on the same day of fever or the next day.

All patients underwent X-ray of the chest and low-dose computer tomography (LDCT) examinations. Chest X-ray was taken using at least one projection depending on the patient age (AP or PA). Diagnostic imaging findings and initial diagnosis were compared with the final diagnosis based on further examinations.

CT examination images were taken with a 128 raw Somatom Definition AS+ (Siemens Healthcare GmbH, Erlangen, Germany) scanner at $120 \mathrm{KV}$ and changeable (depending on patient size) current at mAs values of 25, 50, 75 and 100. The images were reconstructed with a slice thickness of 0.6 and $1.25 \mathrm{~mm}$ for each mAs value. A contrast agent (Ultravist or Omnipaque) was administered with a constant speed of $1.5 \mathrm{~mL} / \mathrm{s}$ and total volume $1 \mathrm{~mL} / \mathrm{kg}$ body weight before the scanning. CT images were assessed by an experienced chest radiologist. To reduce the radiation dose, iterative protocol SAFIRE 3 was carried out.

For CT examination, total dose length product (DLP) ranged from 44 to $150 \mathrm{mGy}^{*} \mathrm{~cm}$ (estimated effective dose 
Table 1. Types on oncologic diseases in the study group

\begin{tabular}{|c|c|c|c|}
\hline Malignancy & No patients (n) & $\operatorname{Sex}(m: f)$ & Mean age \\
\hline ALL & 48 & $31(34.8 \%): 17(34.7 \%)$ & $6.6(m-6.7 ; f-6.5)$ \\
\hline AML & 31 & $17(19.1 \%)$ : $14(28.6 \%)$ & $10.2(m-7.9 ; f-13.1)$ \\
\hline NBL & 26 & $16(18.0 \%): 10$ (20.4\%) & $2.8(m-3.0 ; f-2.5)$ \\
\hline $\begin{array}{l}\text { Ewing } \\
\text { sarcoma }\end{array}$ & 11 & $9(10.1 \%): 2(4.1 \%)$ & $12.1(m-11.5 ; f-15.2)$ \\
\hline $\mathrm{HD}$ & 10 & $7(7.9 \%): 3(6.1 \%)$ & $13.2(m-12.4 ; f-15.1)$ \\
\hline $\mathrm{NHL}$ & 7 & $5(5.6 \%): 2(4.1 \%)$ & $10.2(m-10.2 ; f-10.3)$ \\
\hline RMS & 3 & $2(2.3 \%): 1(2.0 \%)$ & $16.6(m-16.5 ; f-17.0)$ \\
\hline PNET & 1 & $1(1.1 \%): 0$ & $m-10.6$ \\
\hline CML & 1 & $1(1.1 \%): 0$ & $m-12.3$ \\
\hline
\end{tabular}

$A L L$ - acute lymphocytic leukemia; $A M L$ - acute myelogenous leukemia; NBL - neuroblastoma; HD - Hodgkin disease; NHL - non-Hodgkin lymphoma; RMS - rhabdomyosarcoma; PNET - peripheral primitive neuroectodermal tumor; $\mathrm{CML}$ - chronic myelogenous leukemia. consent for this study was waived because of the retrospective nature of the study. The study was conducted in accordance with the Declaration of Helsinki.

The obtained CT results were compared with findings in the plain films and with the overall patient status. If the patient had more than one CT scan of the chest, the CT done closest in time to the onset of the febrile episode and diagnostic microbiological tests was chosen for analysis. The final diagnosis of pulmonary infection was based on bronchoalveolar lavage (BAL) cultures, sputum, blood culture or response to specific treatment (e.g. cotrimoxazol therapy in the case of PCP infections).

The data was collected and analyzed. To compare CT with the plain film results, a t-test for paired samples was carried out. Summary values were given as percentages and a mean.

The criteria for statistical significance were set at $\mathrm{p}<0.05$. Statistical analysis was carried out with STATISTICA software v. 10 (StatSoft, Tulsa, USA).
Table 2. Final diagnosis in 116 (84\%) patients with abnormal results of low dose computed tomography

\begin{tabular}{|l|c|}
\multicolumn{1}{|c|}{ Final diagnosis } & Number of patients (\%) \\
\hline Bacterial infection & \\
Group A b hemolytic streptococci & $26(22.41)$ \\
Coagulase positive staphylococci & $22(18.97 \%)$ \\
Klebsiella pneumoniae & $1(0.86 \%)$ \\
Pseudomonas aeruginosa & $2(1.72 \%)$ \\
Pneumocystis jiroveci & \\
Fungal infection & $8(6.90 \%)$ \\
Aspergillus fumigatus & $14(12.07 \%)$ \\
Candida albicans & $1(0.86 \%)$ \\
Viral infection & \\
Cytomegalovirus & \\
Herpes simplex virus & $3(2.59 \%)$ \\
Mycobacterium tuberculosis & $2(1.72 \%)$ \\
Mycobacteriosis & $3(2.59 \%)$ \\
Unknown etiology & $3(2.59 \%)$ \\
\hline
\end{tabular}

0.748-2.55 $\mathrm{mSv}$ ) depending on the patient's size, while for $\mathrm{X}$-ray examination it was from 0.08 to $0.1 \mathrm{mSv}$. Thus, radiation dose was lower for the X-ray, but its sensitivity in detecting lung lesions and the possibility of their characteristics was considerably limited.

The study was approved by the Commission of Bioethics at Wroclaw Medical University. Written informed

\section{Results}

\section{Chest X-ray findings}

In the study group, 80 (57.97\%) patients had normal X-ray of the chest, while $58(42.03 \%)$ presented abnormalities such as air-space consolidations $(\mathrm{n}=29 ; 21.01 \%)$, nodules ( $\mathrm{n}=4 ; 2.89 \%)$, cavitations $(\mathrm{n}=2 ; 1.44 \%)$, reticulonodular shadows $(\mathrm{n}=3 ; 2.17 \%)$, bronchiectasis $(\mathrm{n}=3$; $2.17 \%$ ), pleural effusion ( $\mathrm{n}=14 ; 10.14 \%$ ), and lymphadenopathy ( $\mathrm{n}=3 ; 2.17 \%$ ). Of the patients with a normal chest radiograph, subsequent $\mathrm{CT}$ of the chest showed pulmonary abnormalities in 58 patients.

\section{CT findings}

CT detected pulmonary abnormalities in 116 (84.06\%) of the 138 patients, such as ground glass opacity (GGO) ( $\mathrm{n}=79 ; 57.25 \%)$, nodules $(\mathrm{n}=60 ; 43.48 \%$ ), and air-space consolidations ( $\mathrm{n}=58 ; 42.03 \%)$. Examples of abnormalities found on CT images are presented in Fig. 1, 2, 3 and 4.

To compare the CT and plain film results, examinations with the findings suggestive of the inflammatory process were analyzed. A t-test for paired samples revealed a statistically significantly higher sensitivity for the relevant chest findings of CT in comparison to plain film, $\mathrm{p}<0.001$.

\section{Final diagnosis}

Bacterial pneumonia was suspected on the basis of radiological findings in 81 patients. It was confirmed in 
Fig. 1. A 12-year-old female patient with febrile neutropenia (ANC $=315 / \mathrm{pL})$. Axial and coronal $\mathrm{CT}$ scans reveal a nodule $(15 \mathrm{~mm}$ in diameter) in the basal segment of the left lung. The nodule is surrounded by a halo of ground-glass attenuation, typical for angioinvasive aspergillosis

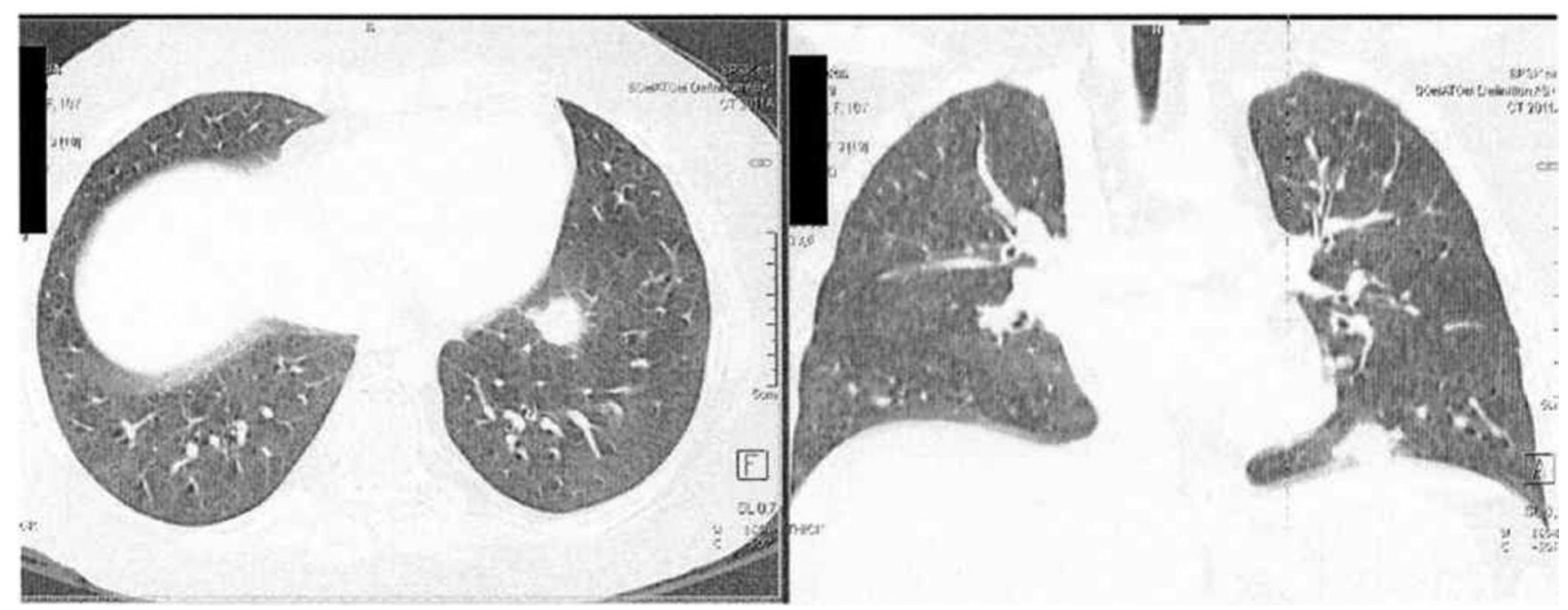

Fig. 2. A 17-year-old male patient with febrile neutropenia 45 days after bone marrow transplantation. Axial CT scan, sagittal and coronal reconstruction show atelectatic changes and large areas of lung tissue consolidation mostly in the left lung. Fluid is visible in the left pleural cavity (16 HU)
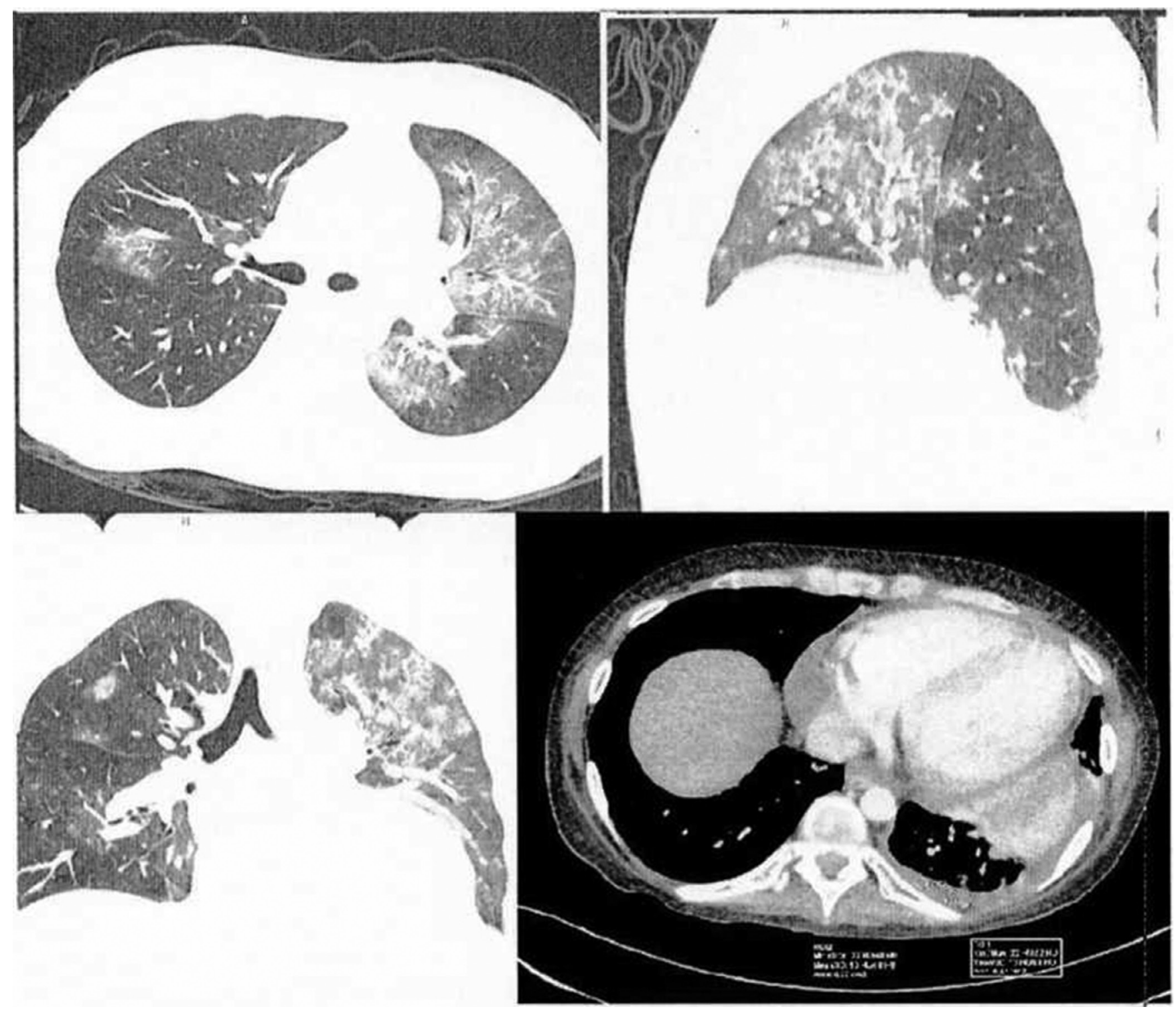
Fig. 3. A 13-month-old male patient with febrile neutropenia. CT scan shows large areas of lung tissue consolidation with partial air bronchogram within large and medium bronchi consistent with pneumonia. Small bronchi cannot be seen - alveolar bleeding cannot be excluded. Image blurring due to motion artifact
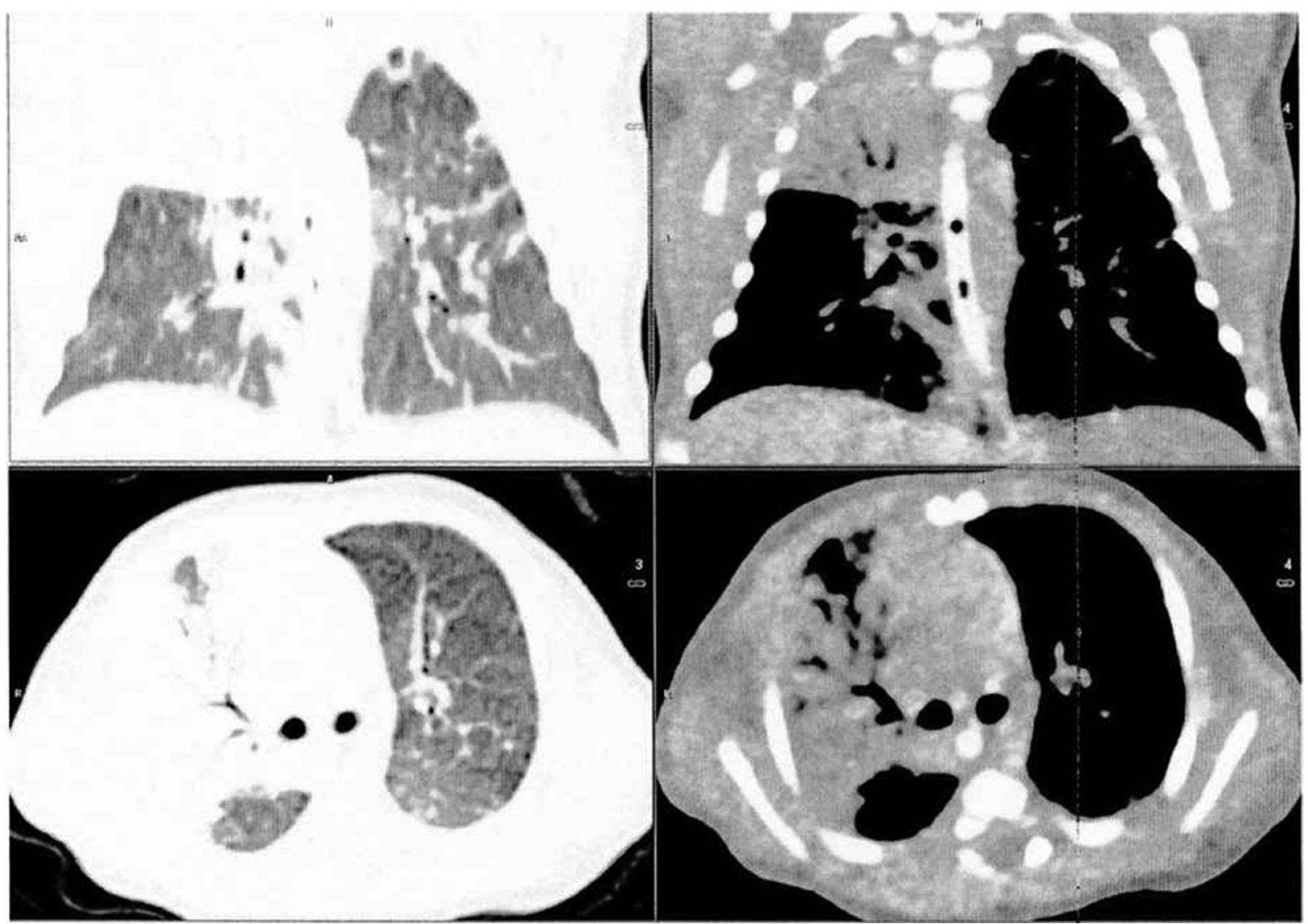

Fig. 4. A 13-year-old male patient with febrile neutropenia ( $\mathrm{ANC}=430 / \mathrm{pL})$. Axial CT scan shows multiple areas of ground glass opacities. Patient presented with persistent cough and chest pain
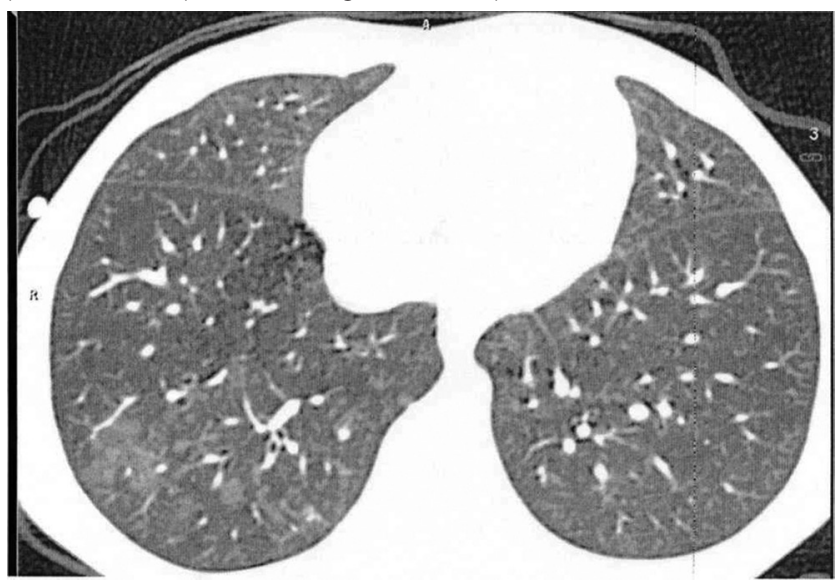

microbiological tests (sputum, BAL, blood cultures) in 51 patients. Positive response to the antibacterial treatment was considered as confirmation of bacterial infection and it occurred in 20 patients. The fungal etiology of pulmonary changes revealed in $\mathrm{CT}$ examination was suspected in 16 patients. It was confirmed by mycology and/or molecular tests in 15 cases. In 3 additional cases, positive response to antifungal treatment was observed. Cytomegalovirus pneumonia and HSV pneumonia were suspected in 6 and confirmed by BAL in 5 patients. Tuberculosis was suspected in 3 cases and in both confirmed in lung biopsy (transbronchial or surgical). Pneumocystis jirovecii pneumonia occurred in 8 patients. Among febrile neutropenic patients with no radiological findings in the lungs, foci of infection were identified in other organs (abdomen, brain, nasal sinuses, etc.) in 13 patients. No potential cause of high fever was identified in 5 patients and classified as unknown etiology. Using LDCT, radiologists were able to correctly characterize the infective lesions in 94 out of 116 patients $(81.03 \%)$. The summary of final diagnoses is presented in Table 2.

\section{Discussion}

Pulmonary complications are common in patients with febrile neutropenia and underlying cancer disease. In patients with acute leukemia, pneumonia develops in 17-24\% of patients. ${ }^{9-11}$ Tenholder and Hooper reported pulmonary complications in up to $98 \%$ of leukemic patient autopsies. ${ }^{12}$ 
Symptoms from the respiratory tract require urgent attention, because the mortality rate due to pulmonary complications reaches $38 \%$ in these high-risk patients. ${ }^{9}$

The high incidence of pulmonary complications in patients with febrile neutropenia indicates that an initial examination carried out with the aim of finding the site of infection should focus on the lungs. Additionally, the poor prognosis in hematologic patients with pulmonary complications that develop during chemotherapy-induced neutropenia has been reported by many authors. ${ }^{10}$ Many of these patients are also in poor condition, which makes it impossible to perform invasive diagnostic examinations; therefore, X-ray of the chest and CT imaging are frequently used during initial diagnosis.

In children with febrile neutropenia and respiratory signs and symptoms, early detection of a focus of infection and its characterization is important for further management; however, the sensitivity and specificity of radiographic examinations can vary and remain unsatisfactory. Heussel et al. showed the presence of pulmonary lesions on CT scans in more than $50 \%$ of patients with febrile neutropenia who had normal chest radiographs. ${ }^{13}$ Donowitz et al. reported abnormal findings on chest X-rays in only $22 \%$ children and Kang et al. in $31.74 \%$ subjects with febrile neutropenia and hematological malignancies. ${ }^{14,15} \mathrm{CT}$ can also detect pneumonia earlier than chest X-ray in children with pulmonary infections. ${ }^{3}$ Therefore, $\mathrm{CT}$ is often performed in patients with febrile neutropenia and normal or questionable chest radiography findings. In our study group of 138 patients with febrile neutropenia, X-ray of the chest revealed abnormalities in 58 (42.03\%) children, while simultaneously performed CT detected pulmonary abnormalities in 116 (84.06\%) of them.

The value of $\mathrm{CT}$ in fast and reliable diagnosis in pediatric patients with neutropenia is undisputed. However, the method has its cons and the most important is radiation. Since the discovery of the harmful effects of using $\mathrm{X}$-ray imaging, researchers have started to optimize the image quality along with minimizing the radiation dose. The concept of ALARA was developed on this premise, which has made it possible to achieve images adequate for diagnostic purposes and keep the radiation dose as low as reasonably achievable. Protecting patients from the risks of using X-ray imaging is especially important in children, who are more susceptible than adults. Children have a higher risk of developing cancer due to radiation exposure because of longer life expectancy than in adults, and more frequent cell division, which makes them more sensitive to radiation. Thankfully, the development of CT scanners and using iterative protocols lead to a significant reduction of the radiation dose. Using a modern 128 raw CT scanner with iterative protocol SAFIRE 3 made it possible to reduce the total dose in comparison with the total dose delivered during X-ray examination.

Another issue is using intravenous iodinated contrast agents. They allow the radiologist to assess mediastinal structures in a more detailed way, to depict slightly enlarged lymphatic nodes, diagnose potential mediastinal pathologies (like abscesses) or evaluate the heart and great vessels. Modern contrast agents are considered safe; however, some complications can occur, including anaphylaxis or renal damage. Additionally, the use of iodinated contrast agents leads to an increase in radiation doses. In our study, none of the examined patients had crucial findings in the mediastinum. Enlarged mediastinal lymphatic nodes were reported in 3 patients; however, these findings have not influenced the final diagnosis, which was established due to lung examination.

\section{Conclusions}

LDCT is an excellent modality in the diagnostic algorithm in pediatric patients with febrile neutropenia. It allows early detection and detailed characterization of pulmonary abnormalities. CT is the most sensitive technique in detecting and describing parenchymal disease. Understanding the CT appearance of these lesions enables an accurate diagnosis and optimizes management of pediatric patients with febrile neutropenia. Moreover, due to unsatisfying plain film sensitivity, it looks reasonable to abandon this method in diagnosing pediatric patients with neutropenic fever. This strategy will make it possible to avoid unnecessary radiation and lead to a shortened time to first diagnosis in CT. Performing CT scan without IV contrast administration, to avoid its side effects and to reduce radiation dose, should be recommended in patients with neutropenic fever.

\section{References}

1. Lehrnbecher T, Phillips R, Alexander S, et al. Guideline for the management of fever and neutropenia in children with cancer and/or undergoing hematopoietic stem-cell transplantation. J Clin Oncol. 2012;30:4427-4438.

2. Weycker D, Barron R, Kartashov A, Legg J, Lman GH. Incidence, treatment, and consequences of chemotherapy-induced febrile neutropenia in the inpatient and outpatient settings. J Oncol Pharm Pract. 2014;20:190-198.

3. Polverosi R, Guarise A, Balestro E, Carloni A, Dalpiaz G, Feragalli B. High-resolution $C T$ of nontuberculous mycobacteria pulmonary infection in immunocompetent, non-HIV-positive patients. Radiol Med. 2010;115:191-204.

4. Torres JP, De IM, V, Kors L, et al. Respiratory viral infections and coinfections in children with cancer, fever and neutropenia: Clinical outcome of infections caused by different respiratory viruses. Pediatr Infect Dis J. 2016;35:949-954.

5. Prasad SR, Wittram C, Shepard JA, McLoud T, Rhea J. Standard-dose and 50\%-reduced-dose chest CT: Comparing the effect on image quality. AJR Am J Roentgenol. 2002;179:461-465.

6. Pearce MS, Salotti JA, Little MP, et al. Radiation exposure from CT scans in childhood and subsequent risk of leukaemia and brain tumours: A retrospective cohort study. Lancet. 2012;380:499-505.

7. Brenner DJ, Hall EJ. Computed tomography - an increasing source of radiation exposure. N Engl J Med. 2007;357:2277-2284.

8. Grant K, Raupach R. SAFIRE: Sinogram Affirmed Iterative Reconstruction. Last updated: 2012. Access date: 07.07.2016. Available from: http://imaging.ubmmedica.com/all/editorial/diagnosticimaging/pdfs/SAFIRE.pdf. 
9. Rossini F, Verga M, Pioltelli $\mathrm{P}$, et al. Incidence and outcome of pneumonia in patients with acute leukemia receiving first induction therapy with anthracycline-containing regimens. Haematologica. 2000,85;1255-1260.

10. Maschmeyer G, Link H, Hiddemann W, et al. Pulmonary infiltrations in febrile patients with neutropenia. Risk factors and outcome under empirical antimicrobial therapy in a randomized multicenter study. Cancer. 1994,73:2296-2304.

11. Schelenz S, Giles D, Abdallah S. Epidemiology, management and economic impact of febrile neutropenia in oncology patients receiving routine care at a regional UK cancer centre. Ann Oncol. 2012;23:1889-1893.

12. Tenholder MF, Hooper RG. Pulmonary infiltrates in leukemia. Chest. 1980;78:468-473.

13. Heussel CP, Kauczor HU, Heussel GE, et al. Pneumonia in febrile neutropenic patients and in bone marrow and blood stem-cell transplant recipients: use of high-resolution computed tomography. J Clin Oncol. 1999;17:796-805.

14. Donowitz GR, Harman C, Pope T, Stewart FM. The role of the chest roentgenogram in febrile neutropenic patients. Arch Intern Med. 1991;151:701-704.

15. Kang M, Deoghuria D, Varma S, Gupta D, Bhatia A, Khandelwal $\mathrm{N}$. Role of HRCT in detection and characterization of pulmonary abnormalities in patients with febrile neutropenia. Lung India. 2013;30:124-130.

16. Heussel CP, Kauczor HU, Ullmann AJ. Pneumonia in neutropenic patients. Eur Radiol. 2004;14:256-271.

17. Kang EY, Patz EF, Jr., Muller NL. Cytomegalovirus pneumonia in transplant patients: CT findings. J Comput Assist Tomogr. 1996;20:295-299.

18. Moon JH, Kim EA, Lee KS, Kim TS, Jung KJ, Song JH. Cytomegalovirus pneumonia: high-resolution CT findings in ten non-AIDS immunocompromised patients. Korean J Radiol. 2000;1:73-78.

19. Franquet T, Lee KS, Muller NL. Thin-section CT findings in 32 immunocompromised patients with cytomegalovirus pneumonia who do not have AIDS. AJR Am J Roentgenol. 2003;181:1059-1063.
20. Demirkazik FB, Akin A, Uzun O, Akpinar MG, Ariyurek MO. CT findings in immunocompromised patients with pulmonary infections. Diagn Interv Radiol. 2008;14:75-82.

21. Reichenberger F, Habicht JM, Gratwohl A, Tamm M. Diagnosis and treatment of invasive pulmonary aspergillosis in neutropenic patients. Eur Respir J. 2002;19:743-755.

22. Grewal P, Brassard A. Fact or fiction: Does the non-HIV/AIDS immunosuppressed patient need Pneumocystis jiroveci pneumonia prophylaxis? An updated literature review. J Cutan Med Surg. 2009;13:308-312.

23. Oh YW, Kim YH, Lee NJ, et al. High-resolution CT appearance of miliary tuberculosis. J Comput Assist Tomogr. 1994;18:862-866.

24. Stranzinger E, Schindera S, Cullmann J, Herrmann R, Schmitz S, Wolf R. Pediatric CT of the lung: Influences on image quality. Open Journal of Radiology. 2013;3:45-50.

25. Strauss KJ, Goske MJ, Kaste SC, et al. Image gently: Ten steps you can take to optimize image quality and lower CT dose for pediatric patients. AJR Am J Roentgenol. 2010;194:868-873.

26. Han BK, Grant KL, Garberich R, Sedlmair M, Lindberg J, Lesser JR. Assessment of an iterative reconstruction algorithm (SAFIRE) on image quality in pediatric cardiac CT datasets. J Cardiovasc Comput Tomogr. 2012;6:200-204.

27. Wang R, Schoepf UJ, Wu R, et al. CT coronary angiography: image quality with sinogram-affirmed iterative reconstruction compared with filtered back-projection. Clin Radiol. 2013;68:272-278.

28. Yu L, Fletcher JG, Shiung M, et al. Radiation dose reduction in pediatric body ct using iterative reconstruction and a novel imagebased denoising method. AJR Am J Roentgenol. 2015;205:1026-1037.

29. Baumueller S, Hilty R, Nguyen TD, Weder W, Alkadhi H, Frauenfelder T. Influence of sinogram-affirmed iterative reconstruction on computed tomography-based lung volumetry and quantification of pulmonary emphysema. J Comput Assist Tomogr. 2016;40:96-101.

30. Pontana F, Billard AS, Duhamel A, et al. Effect of iterative reconstruction on the detection of systemic sclerosis-related interstitial lung disease: Clinical experience in 55 patients. Radiology. 2016;279:297-305. 